\title{
Wave/Particle Duality: The La Brea Tar Pit of Quantum Theory
}

\author{
Paul A. Klevgard
}

\begin{abstract}
Why do photons and speeding electrons have both wave features and particle features when common sense tells us that they should be either particle or wave and not an amalgam of both? Part I of this paper deals with photons and argues that there are flaws in the assumptions we have made regarding their particle nature. The argument depends upon distinguishing between two identities of the photon, namely unstored energy and its stored (relativistic) mass. Part II extends these arguments to the case of the speeding electron and argues that current ontological assumptions made about projectiles have exacerbated our confusion about the nature of moving electrons. When regarded ontologically, projectile motion is not as simple as has been assumed by both classical and modern physics.
\end{abstract}

Key words: duality, quantum mechanics, particle, wave, realism, projectile motion, ontology, photon, objective indefiniteness

\section{PART I}

\section{INTRODUCTION}

How can a speeding electron be both a particle and a wave if their properties are incompatible? For some eight decades now, we have been stuck with this question, and progress toward its solution has been, to be charitable, disappointing. The Copenhagen approach argues that we have reached the terminus of human understanding and that a little humility on our part is now in order since the essence of reality is beyond our ken. But humility among philosophers, and even among physicists, is always in short supply. And, happily, in the past, setting out limits on human knowledge has always proved dangerous. The mere attempt to set limits on our understanding rubs some people the wrong way. Einstein is a prime example.

There have been many attempts over the years some in these pages - to resolve the wave/particle conundrum, but few approaches have gained much acceptance. Typically such explications come with the baggage of postulating new, hypothetical entities or effects for which there is no hard evidence. One approach to reconciling wave with particle that has gained some support was taken by David Bohm. ${ }^{(1)}$ His ideas have attracted interest over the years because he reasserted the principle that physical entities have a defined reality independent of their observer or their measurement. Bohm's ontology was largely classical, and his assumptions were those of realism. Particles had a distinct, if immeasurable, location and their relationship to the quantum potential was causal in the traditional sense. Starting with the Schrödinger wave equation, Bohm refactored it to yield a term that, he argued, could explain the strange behavior of the speeding electron. His arguments were well founded in terms of logic and mathematics, although Bohm later admitted that his quantum potential was "rather strange and arbitrary."(2) It seems evident, however, that Bohm determined his destination (classical physics, realism) before he began his inquiry, which makes his theory appear contrived. But that does not diminish the importance of his contribution. Bohm's theory served to refocus attention on the ontological questions hidden by the formalism of quantum mechanics. An ontological inquiry may still serve us well, providing we avoid the temptation of predetermining our destination.

\section{ROAD MAP}

This short essay will examine the wave/particle duality problem for two separate entities, the photon, which has no rest mass, and the high-speed electron, or similar particle, which has rest mass. The common approach toward both entities will be an ontological analysis. In his last book Bohm asserts that "Ontology is concerned primarily with that which is and only secondarily with how we obtain our knowledge about this." ${ }^{(3)}$ But defining ontology as merely the study of that which exists seems unduly restrictive when dealing with physics, so I shall instead extend the 
term to cover mass/energy entities that either exist (as matter) or occur (as radiation). My use of "ontology” and "ontological" in what follows is grounded in physics and is intended to be straightforward: the terms are shorthand for the nature of existence/ occurrence, and relating to existence/occurrence, respectively. No hypothetical entities will be advanced or allowed in this analysis: no pilot waves, no messenger waves, no multidimensional time, etc.

In addition to an ontological inquiry, I propose to examine the photon and the electron from the vantage point of form (wave, field) rather than content. This gives us three guiding requirements for our little journey: an ontological viewpoint, entities of mass-orenergy, and the latter regarded formally. These conceptual markers should lead us to our first rest stop and perhaps beyond.

\section{QUANTA}

Both classical and modern physics place great emphasis on massy objects (i.e., particles) moving in space over time. As we shall see, such projectile motion is absolutely the wrong place to start for an ontological analysis of physics. With the reader's indulgence, I will begin by limiting our consideration to nonprojectiles, i.e., massy objects that are stationary in space relative to some observer.

With that in mind, consider the following formal comparison between mass quanta and energy quanta. We know that mass-as-stationary-matter exists, that it is quantized, that it has the field form, and that it progresses (ages) over time. We also know that energy-as-radiation occurs, that it is quantized, that it has the wave form, and that it progresses over space. In physics this type of formal comparison of molecular quanta with radiation quanta has a long and honorable history. ${ }^{1}$

Ever since Faraday, physics has employed the concept of field. In its original formulation it was stored energy (e.g., electric charge) existing in space that could causally affect remote particles. Whether the stored energy was electric, magnetic, or gravitational was irrelevant to the field as a formal concept. Because all varieties of energy have mass, as Einstein argued in 1905, it is equally valid to describe the field as mass distributed over space. If that mass takes the form of matter (e.g., molecules), then we have a scalar density matter field. From the ontological point of view an electric field and a molecular gas (or solid) confirm that different quanta can share a common form whose ontological characteristics are (1) existence, (2) extension in space, and (3) being at rest in space relative to some observer. Fields have a massover-space rate, i.e., density, just as photons have an energy-over-time rate, i.e., power.

To continue with our formal comparison, both mass and energy may become potential and be stored by their opposite number. Specifically, potential energy may be strain for the watch spring, thermal motion for the molecular gas, or an electrostatic potential for an individual molecule. All constitute stored, potential energy. Meanwhile, potential mass is simply the relativistic mass of a projectile or a photon courtesy of their respective kinetic energies and $E=m c^{2}$. Simply put, a potential entity is stored mass or energy that adopts the field-or-wave form of the quantum that stores it.

Whether potential energy is mechanical strain, heat, or electric charge, that energy exists and is spacedistributed. This means that potential energy, like space-stationary matter, has the field form.

But what about potential, i.e., relativistic, mass? Is it really a formless scalar, as physicists tend to assume? Rephrasing the question, is the mass of a photon simply a quantity that has neither form nor a presence in a dimension, whereas the energy of a space-stationary particle is quite the reverse? Both the equality of mass and energy plus their formal parallels as cited above suggest that the answers to these questions is a definite "no."

\section{CHOOSING A RADICAL PATH}

We have reached a fork in the road and must choose a path. Shall we stick with the traditional view of quantitative relativistic mass? Or shall we assume that mass and energy will always have a form, either field or wave? We know that always following the traditional path will lead us straight toward the creosote, so let's make our break with tradition here and hope for the best. Both analysis and symmetry suggest that mass and energy can never exist or occur as scalars; they must always have the field form (e.g., matter motionless in space for an observer), or the wave form (electromagnetic radiation), or a mixture of the two (projectiles).

If that is the case and we are correct in assuming that the space-stationary particle is a field of kinetic mass and potential energy, then the radiation quantum (photon) must be a wave of kinetic energy and potential mass. If this is true, then equality of wave/ field form is restored to both the kinetic and potential sides of mass and energy. This conclusion has an analytic and a heuristic benefit in that whatever applies to the existing/mass/space side of reality 
should have a formal counterpart in the occurring/ energy/time side of reality. If we cannot find such formal counterparts, then surely we have taken the wrong path. This requirement that existing mass have a formal parallel in occurring energy may be called the radical equality of mass and energy.

Asserting that the photon is a wave of kinetic energy and potential mass implies that $E=m c^{2}$ always has a kinetic quantity on one side of the equal sign and a potential, stored quantity on the other side. That is how $E=m c^{2}$ should always be interpreted. Hence a photon of kinetic energy $e_{o}$ has a potential mass of $e_{o}$ $\div c^{2}$ and a space-stationary particle of kinetic mass $m_{o}$ has a potential energy of $m_{o} \times c^{2}$.

A photon progresses over a dimension (space) at the maximum possible rate, namely the speed of light. Photons are also stationary in the opposite dimension (time), as we know from special relativity. This suggests that the photon is delimited (discrete) in time and located there as well, relative to an idealized time-reference photon. The formal equivalent of this is our space-stationary particle, which is delimited (discrete) in space and located there as well, relative to some space-reference particle/object. Like a photon, a space-stationary particle also progresses in a dimension, namely time, at the maximum possible rate. $^{2}$

Stored (potential) energy as an existing field progresses over time, and since the temporal dimension is linear, stored energy never attenuates. Stored (potential) mass as an occurring wave progresses over space, and since the latter is three-dimensional, stored mass will attenuate as the wave-front spatially expands. Rather than violating the formal relationship between the two cases, this difference in the behavior of stored energy and mass as they progress in their respective dimensions merely reflects the differences of space versus time.

Any potential mass or any potential energy assumes the form of its associated kinetic entity; potential energy stored by matter assumes matter's timeprogressing field form while potential mass stored by radiation assumes radiation's space-progressing/ expanding wave form. This is why the photon's stored mass is truly continuous over all of the photon's space paths. The photon's stored/potential mass may be infinitely rarefied over space while its orthogonal kinetic energy identity (oscillation) remains unaffected. This behavior exemplifies a rule that holds for all ontological entities: progression of the potential identity leaves the kinetic identity unaffected. An obvious example is the existing, space- stationary particle whose kinetic mass (matter quantum) is unaffected by its progression over time.

Potential mass and potential energy progressing over time and space, respectively, will at some point cease to be stored, which is to say it will become kinetic. An atom or molecule may store energy when one of its inner electrons temporarily moves to an outer shell. At some point in its time progression this electron will relocate and its potential energy will be released as kinetic energy (a photon). Similarly, a photon, which stores mass, at some point in its space progression may impinge upon matter and thereby release its potential mass as kinetic mass.

The release of stored energy by a particle is called "emission," and the release of stored mass by a photon is called "absorption." The two processes are formally identical. Both constitute crossover between the realms of radiation and matter: mass or energy stored in one form, wave or field, is released as kinetic in its opposite form, field or wave. We use crossover to gain information about entities, including those we cannot measure directly. Unfortunately, physicists have not been consistent in their interpretation of crossover measurement.

\section{CROSSOVER MEASUREMENT}

Suppose you are in a dark room and wish to characterize a large, stationary, massy object, say, a warm bowling ball. You can pick it up with your hands and determine that it has rest mass and extends over space. Ontologically, you would say that the entity exists and has the field form. You can also put the bowling ball down, hold your hands three inches away from the ball, and feel its infrared radiation in your palms and fingers. But you would not presume that the object was itself radiation and wave-like simply because it had stored field energy that it was releasing in wave form via crossover.

So far, so good. But a bowling ball is a matter field of kinetic mass, and its ontological opposite is a radiation wave of kinetic energy. If you have radiation of one or more photons to measure, you might arrange for the photon(s) to wave-diffract through a narrow slit or prism and observe the dispersion path(s). From this you might infer that photons are radiation energy that occurs (wave oscillation). But what if you go further and emulate P.N. Lebedev (1910) to demonstrate that a photon absorbed by a material body gives up a small but measurable momentum at a space point, exactly as a particle does? One of your experiments would indicate that the photon is a wave and the other experiment that it 
is a particle.

At this point, you have two choices. Choice one leads through Copenhagen and requires you to admit that the photon exhibits, depending upon experiment, two contradictory identities, and its true nature can never be known. You have arrived at the tar pit.

Choice two is to accept the radical equality of mass and energy and argue that both mass quanta and energy quanta have kinetic and potential identities the measurement of which yields ontologically opposite (field/wave) entities. Nothing requires us to take the measurement results of both the kinetic and potential identities of a quantum and treat them as together defining the nature of but one identity of one entity. The existing field identity of a bowling ball is not put in question by the release of its stored energy as kinetic energy waves via emission crossover. By the same token, the occurring wave identity of the photon is not compromised by the release of its stored (relativistic) mass as kinetic mass field via absorption crossover.

Another way to look at this is that quanta interact with like quanta via their kinetic identities, while their potential identities are involved only in crossover to the opposite realm. Thus bowling balls interact with each other as kinetic mass field quanta, while their potential energy remains locked away until released to the radiation realm. Similarly, photons interact with each other (diffraction, reinforcement) as kinetic energy wave quanta, while their potential mass remains locked away until released to the realm of matter.

\section{THE PHOTON IS NOT A PARTICLE}

After the photon emerged as a new quantized entity within physics, and it became apparent that it progressed over space, it was inevitable that the particle concept (an existing, space-located, massy entity) would be used to describe the photon. This conceptual model of a moving, existing matter quantum as a projectile progressing over space had always dominated physics since it accords with common sense and the classical view of entities.

Of course, slight problems were bound to emerge from this intellectual leap. It was puzzling that waves followed many space paths while particles were presumed to follow only one. But bright minds were ingenious and these things could be explained, or at least explained away. As a result, "particle" became an amorphous concept in quantum theory. The word now denotes a pseudo-entity whose attributes are essentially incompatible: existing yet occurring, space-located yet space-smeared, field-like yet also wave-like. Unmeasured, the "particle" is all these things, but once measured it conforms to a mere subset and we who do the measuring will have forced an unknowable reality into categories that we can comprehend. This approach surely merits the description of "explaining away."

Such contorted logic can be avoided if we accept the fact that the photon is simply an occurring energy wave with kinetic and potential identities. To be sure, the proposition that the photon is not a particle before crossover and, hence, does not suffer from wave/ particle duality must contend with two obstacles.

First is people's mental inertia. Acceptance of this new concept requires us to unlearn something we thought we knew, and that is always hard. Fortunately, there is an advantage the new concept brings us and that is a formal correspondence between all quanta regarding how we should interpret measurements of their kinetic and potential identities. If an entity's kinetic identity displays the wave form, the entity's potential identity will (upon release) display the field form and vice versa.

A second obstacle is the fact that photons are not alone in their exhibition of both wave and particle characteristics. Hence dismissing the "particle" behavior of photons as a crossover artifact does not eliminate duality per se. If we are to come to grips with the wave/particle conundrum, we are forced to resolve it for projectiles - speeding quantum entities with rest mass - and not merely for photons lacking rest mass. We'll undertake that task in Part II of this essay.

\section{PART II}

\section{WAVES WITH REST MASS}

The wave/particle duality of projectiles is incontrovertible. Projectiles really do have two dissimilar identities. Our task then is to provide an ontological basis for the dual nature of projectiles that both exist and occur, that are both wave and field. Toward that end, we may now remove our restriction on particle motion to consider the case of a particle moving through space relative to an observer. What ontological changes result from particle motion? And can we expect similar changes from photon "motion?" If not, the formal equality of mass and energy is in trouble and once again the tar pit is just around the corner.

Fortunately, formal equality is not about to fail us. A particle (material object) moving relative to an observer possesses kinetic energy relative to that 
observer. Adding kinetic energy to a space-stationary particle has its ontological counterpart in adding kinetic mass to a time-stationary photon. Both additions create a projectile, and a projectile is a wave/ field entity distinct from either a space-stationary particle (field only) or a time-stationary photon (wave only). Obviously, adding kinetic energy to a spacestationary particle is easier to accomplish in the laboratory than is adding kinetic mass to a photon; nevertheless, the two additions are formally identical. Once a photon acquires kinetic mass and ceases to be a photon, its space velocity is diminished, since no entity with rest mass travels at the speed of light. This rest-mass-possessing, part-field, former-photon is no longer stationary in time, and the more massive it becomes the slower it travels in space and the faster it progresses in time.

Kinetic energy must always have the wave form. This simple statement, if true, is deeply subversive of physicists' current interpretations of entities. It means that kinetic energy, like kinetic mass, is an actual entity that extends in one dimension and progresses in the other. Kinetic energy waves not hosted by material media come in two varieties, electromagnetic waves and de Broglie waves.

Space-stationary particles have no kinetic energy and, therefore, no kinetic energy waves. At the opposite extreme, photons are all kinetic energy and their waves are always electromagnetic in nature. Electromagnetic waves both represent and constitute the kinetic energy of a photon. In between the spacestationary particle and the photon is the projectile with its mixture of kinetic mass and kinetic energy and with waves that were first described by Prince Louis de Broglie in 1924. De Broglie waves both represent and constitute the kinetic energy of a projectile. If a projectile loses its kinetic energy, its de Broglie waves disappear. If a projectile becomes almost entirely kinetic energy (negligible mass, velocity close to that of light), then its de Broglie radiation, without becoming electromagnetic, approaches the velocity of the photon and approximates the photon's wave character.

We may now summarize the ontological characteristics of projectile motion relative to some observer:

a) The projectile has kinetic mass plus kinetic energy, it has the field form plus the wave form, and it progresses through space while also progressing through time.

b) The projectile must have these dual characteristics, and those entities that lack them constitute the natural limits of projectile motion: the fieldform, space-stationary, wave-bereft particle on one side, and the wave-form, time-stationary, field-bereft photon on the other side.

c) Projectiles that are near the photon limit of motion for an observer are space-fast and time-slow, and wave form and occurrence dominate field form and existence for that observer. Projectiles that are far away from the photon limit of motion for an observer are time-fast and space-slow, and field form and existence dominate wave form and occurrence for that observer.

\section{UNCERTAINTY AND OBJECTIVE IN- DEFINITENESS}

By definition, an existing space-stationary field has a well-defined space position but has no measurable wavelength or momentum value. For an occurring time-stationary wave (photon), just the reverse is true: momentum and wavelength are well defined, but space position is not. If you combine these two states, as projectiles do, then you have a trade-off in measurement precision that we know as the "uncertainty principle." Since the projectile truly is a fusion of kinetic energy wave and kinetic mass field, its position and its momentum are objectively indefinite and not simply obscured by measurement technique. Objective indefiniteness and the wave nature of projectiles are important concepts that, unfortunately, have often been misinterpreted.

Objective indefiniteness has been cited as proof that philosophical realism is both wrong-headed and untenable. These claims are certainly true regarding a naive realism that clings to a classical ontology and an unqualified causal determinism. But they are not true for a more robust realism that is receptive to the concept of projectile kinetic energy as a wave form. Once that concept is accepted, then objective indefiniteness becomes a necessary consequence of combining mass/field/existence with energy/wave/ occurrence to create projectile motion. Within such mixed-modes entities the objective reality of mass and energy remains intact, always a goal of the realist. On the other hand, the realist must abandon strict causal determinism in crossover and accept the ontological validity of the occurring and wave-like side of reality (de Broglie waves, potential mass, etc.).

A second misinterpretation is to trumpet energy and wave as somehow more "real" than mass and field. This position appeals to those who believe that material particles, however tiny, can always be 
subdivided and are ultimately based on energy. It also appeals to those who regard the Schrödinger wave equation as the basis for all our physical knowledge. Needless to say there is no ontological basis for exalting wave/energy over field/existence. The great body of physical knowledge we have points to the equality and interdependence of mass and energy, of field and wave, of existence and occurrence.

\section{CROSSOVER AND CHANCE}

Crossover has been mentioned with regard to the photon, and the role it plays in projectile motion is our last topic. From the perspective of the radical equality of mass and energy, crossover is always potential mass-or-energy stored in one form being released as kinetic energy-or-mass in the opposite form. The projectile is only partially kinetic energy, but what energy it has resides in the multiple de Broglie waves that constitute part of the projectile's identity. By virtue of $E=m c^{2}$, these energy waves have an associated stored (potential) mass also in wave form. These waves of potential mass play a crucial role in crossover.

The multiple de Broglie waves of a projectile interact with (reinforce) each other, both positively and negatively, depending on the nature of the projectile. If the projectile is high in mass and low in velocity, then the wave reinforcement/interaction is extensive and potential mass constitutes a well-defined field (i.e., wave-packet) coinciding with the kinetic (rest) mass field itself. The release of this potential mass, which renders it kinetic, occurs at the point of impact of the rest mass against a material barrier. At that point and instant, all of the kinetic energy of the de Broglie waves is transferred to the barrier by the mechanism of the impact/crossover/conversion of the potential mass associated with the de Broglie waves. There is no uncertainty regarding the impact/ crossover point because the potential mass location is precisely defined as it must be when the projectile's rest mass is enormous compared to its potential mass. The potential mass of the projectile is constrained to cross over at the impact location of the dominant rest mass.

If, however, the projectile is minuscule in mass and very high in velocity, then the de Broglie wave reinforcement/interaction is so attenuated that the potential mass resembles a fuzzy wave (a wave whose momentum/wavelength values are somewhat imprecise) rather than a field. In this case (e.g., an electron moving at a speed close to that of light), the potential mass is quantitatively large compared to the kinetic (rest) mass and, being wave-like, is no longer confined or localized in space. But the crossover of this potential mass wave-packet renders it both kinetic and precisely located in space. ${ }^{3}$ This means that while the potential mass of such a projectile is space-smeared to follow all available space paths, crossover (and rest mass impact) actually occurs at the terminus of a single space path. Consequently, the space location of crossover/impact is necessarily probabilistic, and the distribution of the potential mass in space determines the local probability of crossover. The rest mass of the projectile is constrained to impact at the crossover location of the dominant potential mass.

The wave-like potential mass of a projectile is real and not simply a mathematical quantity derived from $E=m c^{2}$. Because such a potential mass is a wave, it travels multiple space paths with the result, for example, that it travels through both slits in the double-slit electron diffraction experiment. And this means, among other things, that we no longer have to suffer such descriptions as "the speeding electron senses the presence of the slit through which it does not travel." The speeding electron's potential mass wave does, in fact, travel through both slits. The concept of potential mass derives from the general principle of the radical equality of mass and energy. If potential energy can exist as a field stored in matter, then potential mass can occur as a wave stored in radiation. Except as a scalar quantity, potential mass has been largely ignored in physics, which is a major reason why radiation and radiation-like projectiles have remained such a mystery to us.

The idea that projectile potential mass occurs as a wave should be viewed within the context of a larger conceptual framework. Whether quanta exist as fields, occur as waves, or do both as projectiles, they have both a kinetic and a potential identity, each with a corresponding form. The form for kinetic mass (matter) is field, and the form for kinetic energy (radiation) is wave. The form for potential (relativistic) mass is wave, and the form for potential energy is field. Once this is understood, wave/particle duality is no longer such a big mystery.

Niels Bohr was a great and profound thinker. Like everyone else in his day, however, he accepted a classical viewpoint that regarded the projectile as ontologically indistinguishable from stationary rest mass. One had movement and a scalar kinetic energy, and the other did not. But these features aside, both stationary rest mass and projectile mass were simple existing objects precisely located in space and in time. When de Broglie's theory and the Davisson-Germer 
experiments proved the classical concept of projectile to be seriously flawed, Bohr took the wrong path. Instead of faulting the common-sense notion of projectile as a simple existent, he instead faulted humankind: wave/particle duality reflected our permanent inability to comprehend the mysteries of nature.

But taking space-stationary rest mass and adding kinetic energy to it does not produce a classical projectile that exists along a trajectory. Instead, it breeds a hybrid beast of both kinetic mass and kinetic energy with the relative proportions of mass and energy determining its form, field or wave. This concept makes wave/particle duality — which technically is wave/field duality — finally comprehensible.

An ontological analysis of mass and energy can guide us further than we have gone so far and allow us to explore other areas. There are, for example, the great questions surrounding nonlocality and quantum entanglement. But climbing these summits is left for another day; this is a good resting point in our journey. We have ascended a small hill that affords us at least a limited perspective of the surrounding landscape of mass and energy organized as field and wave. And there is no tar pit in sight.

Received 26 July 2006.

\section{Résumé}

Pourquoi les photons et les électrons rapides ont-ils des caractéristiques d'ondes et des caractéristiques de particules quand le bon sens nous dit qu'ils devraient être ou bien particules ou bien ondes et pas un amalgame de tous les deux? La partie I de cet article traite des photons et argumente du fait qu'il y a des failles dans les suppositions que nous avons faites concernant leur nature de particules. L'argumentation dépend de la distinction de deux identités du photon, à savoir l'énergie non-accumulée et sa masse (relativiste) accumulée. La partie II prolonge ces arguments au cas de l'électron rapide et argumente du fait que les propositions ontologiques actuelles faites au sujet des projectiles ont aggravé notre confusion au sujet de la nature des électrons mobiles. Une fois considéré ontologiquement, le mouvement de projectile n'est pas aussi simple qu'a été supposé par la physique classique et la physique moderne.

\section{Endnotes}

1 For example, in his 1905 paper on the photoelectric effect, ${ }^{(4)}$ Einstein compared the entropy of molecular gas quanta with that of radiation gas quanta. His conclusion was that radiation and the ideal gas were identical in form and hence radiation was also composed of discrete "particles," namely energy quanta. Between 1905 and 1924, Einstein made numerous other comparisons between molecular quanta and radiation quanta, but only one instance will be cited here. In the summer of 1924, shortly after he forwarded the paper of S.N. Bose for publication, Einstein, stimulated by the ideas of Bose, produced his own paper. ${ }^{(5)}$ While Bose used a new quantumcounting method to derive Planck's law for the radiation gas, Einstein used the same counting procedure to derive a quantum theory of the ideal gas of monatomic molecules. But it was Louis de Broglie who probably made the most well-known formal comparison between radiation quanta and matter quanta. Whereas Einstein made the original wave/particle duality argument regarding radiation quanta in 1909, in 1924 de Broglie suggested that the same duality characterized matter quanta. As he had done with Bose, Einstein lent his considerable weight to the new idea and followed up with his own paper, ${ }^{(6)}$ using de Broglie's ideas to extend the arguments of his preceding (1924) paper. ${ }^{(5)}$ Einstein took the statistical mechanics that Bose used for radiation quanta and applied them to lowtemperature atoms, demonstrating that they applied to matter quanta as well. This established the theory of what we know as the Bose-Einstein condensates. In this paper, Einstein wrote “... the analogy between the gas of (radiation) quanta and the gas of molecules must be complete.”

2 The space-stationary particle moves through time at the maximum rate possible because once the particle starts to move its clocks start to slow down as seen by a "stationary" observer.

3 The potential mass of the moving electron behaves the same as the potential mass of the photon. Such mass becomes kinetic, and therefore space-located, upon crossover ("impact”). 


\section{References}

1. D. Bohm, Phys. Rev. 85, 166, 180 (1952); reprinted in Quantum Theory and Measurement (1987), p. 369.

2. Idem, Wholeness and the Implicate Order (Routledge, New York, 1980), p. 80.

3. D. Bohm and B.J. Hiley, The Undivided Uni- verse: An Ontological Interpretation of Quantum Theory (Routledge, New York, London, 1993), p. 2.

4. A. Einstein, Ann. Phys. 17, 132 (1905).

5. A. Einstein, Sitz. Ber. Preuss. Akad. Wiss. 22, 261 (1924)

6. Ibid. 23, 3 (1925).

\section{Paul A. Klevgard}

1179 Westbury Drive

San Jose, California 95131 U.S.A.

e-mail: pklevgard@uwalumni.com 